\title{
Quenched Spin Tunneling and Diabolical Points in Magnetic Molecules: I. Symmetric Configurations
}

\author{
Anupam Garg* \\ Department of Physics and Astronomy, Northwestern University, Evanston, Illinois 60208
}

(October 24, 2018)

\begin{abstract}
The perfect quenching of spin tunneling that has previously been discussed in terms of interfering instantons, and has recently been observed in the magnetic molecule $\mathrm{Fe}_{8}$, is treated using a discrete phase integral (or Wentzel-KramersBrillouin) method. The simplest model Hamiltonian for the phenomenon leads to a Schrödinger equation that is a five-term recursion relation. This recursion relation is reflection-symmetric when the magnetic field applied to the molecule is along the hard magnetic axis. A completely general Herring formula for the tunnel splittings for all reflection-symmetric five-term recursion relations is obtained. Using connection formulas for a new type of turning point that may be described as lying "under the barrier", and which underlies the oscillations in the splitting as a function of magnetic field, this Herring formula is transformed into two other formulas that express the splittings in terms of a small number of action and action-like integrals. These latter formulas appear to be generally valid, even for problems where the recursion contains more than five terms. The results for the model Hamiltonian are compared with experiment, numerics, previous instanton based approaches, and the limiting case of no magnetic field.
\end{abstract}

75.10Dg, 03.65.Sq, 75.50Xx, 75.45.+j

Typeset using REVTEX 


\section{INTRODUCTION}

In several previous papers [1], we have discussed the tunneling of a spin described by the model Hamiltonian

$$
\mathcal{H}=-k_{2} J_{z}^{2}+\left(k_{1}-k_{2}\right) J_{x}^{2}-g \mu_{B} \mathbf{J} \cdot \mathbf{H}
$$

where $\mathbf{J}$ is dimensionless spin operator with components $J_{x}, J_{y}$, and $J_{z}, \mathbf{H}$ is an external magnetic field, and $k_{1}>k_{2}>0$. Since most of the earlier work has been for the special case where $\mathbf{H} \| \hat{\mathbf{x}}$, let us first limit ourselves to that. Viewed as a function of a classical angular momentum vector $\mathbf{J}$ of fixed length $J$, this Hamiltonian has two degenerate minima for $H_{x}<H_{c}=2 k_{1} J / g \mu_{B}$. On general grounds we expect quantum mechanical tunneling to lift the degeneracy, and split the energies. The surprise is that the ground state tunnel splitting, $\Delta_{0}$, is an oscillatory function of $H_{x}$, vanishing exactly whenever

$$
\frac{H_{x}}{H_{c}}=\frac{\sqrt{1-\lambda}}{J}\left[J-n-\frac{1}{2}\right],
$$

where $\lambda=k_{2} / k_{1}$, and $n=0,1, \ldots, 2 J-1$.

The quenching of $\Delta_{0}$ was first studied purely as a theoretical curiosity, and explained in terms of instantons [1](a). Since then, this effect has been observed [2] in the magnetic molecule $\left[(\operatorname{tacn})_{6} \mathrm{Fe}_{8} \mathrm{O}_{2}(\mathrm{OH})_{12}\right]^{8+}$ (or just $\mathrm{Fe}_{8}$ for short), which is approximately described by the Hamiltonian (1.1), with $J=10$, and $k_{1} \approx 0.33 \mathrm{~K}$, and $k_{2} \approx 0.22 \mathrm{~K}$ [3 [5]. Motivated by a desire to use only elementary methods of analysis, the problem was restudied [1](d) using the discrete phase integral (DPI) method (also known as the discrete Wentzel-KramersBrillouin method). An approximate version of this method was developed and applied to $\mathrm{Fe}_{8}$ independently by Villain and Fort [6]. The results of these later studies confirm Eq. (1.2) for the ground pair quenching points, and also find additional quenching points, as we discuss next.

To help grasp the full richness of the spectrum of the Hamiltonian (1.1), we show in Fig. 1 the results of numerical calculation of the energies as a function of $H_{x}$, for $J=3$, for three different values of $H_{z}$. In all three cases, $H_{y}=0$. In part (a), $H_{z}=0$, and we have the symmetric situation mentioned above. Note that (i) the lowest two energy level curves cross six times (including negative values of $H_{x}$ ), and (ii) the crossing points are perfectly periodically spaced, in complete accord with Eq. (1.2). Wernsdorfer and Sessoli [2] have shown the existence of analogous crossings for $\mathrm{Fe}_{8}$. To quantitatively account for the observed period, one must include higher order anisotropies in the Hamiltonian. This does not change the basic physics. In addition, Fig. 11(a) also shows a number of crossings of higher energy levels, of which the analog in $\mathrm{Fe}_{8}$ has not been seen yet.

In Fig. 1(b), $H_{z}$ has a specific non-zero value. The problem is no longer symmetric, and one of the classical minima is lower than the other. Correspondingly, we see that the lowest quantum mechanical state is always non degenerate. However, ignoring tunneling for the moment, the first excited state in the deeper well can have an energy equal to that of the lowest state in the shallower well if $H_{z}$ is correctly chosen. And indeed, we see from the figure that the second and third energy levels do cross a number of times. These crossings, when $\mathbf{H}$ has an easy component, were not anticipated in Ref. [1](a), and were discovered by Wernsdorfer and Sessoli experimentally. As seen in the experiments, the crossings in 
Fig. 1(b) are shifted by half a period from those in Fig. 1(a). Note that as in part (a), Fig. 1(b) displays crossings between yet higher energy levels (the fourth and fifth, e.g.), which have also not been seen experimentally yet.

This pattern continues as $H_{z}$ is increased still further [Fig. 1(c)]. Now the lowest two levels in the deeper well are nondegenerate, and the lowest crossings are between levels 3 and 4. Comapred to Fig. 1(b), these crossings are shifted by yet another half-period, just as seen experimentally. Again, there are crossings between higher pairs of levels, and again only those between levels 3 and 4 have been seen in $\mathrm{Fe}_{8}$.

It is clearly interesting to understand the structure in the energy spectrum analytically, and numerical diagonalization alone cannot provide this. When $J$ is of order 10 , as it is for $\mathrm{Fe}_{8}$, a semiclassical analysis is natural, and it is profitable to think of the energy differences amongst low lying levels in terms of tunneling. Such an analysis was done in Ref. [1] (a-d). In this paper, we shall elaborate on our earlier DPI analysis [1](d), and provide several results that are more generally applicable to Hamiltonians other than Eq. (1.1). We shall limit ourselves, however, to problems which are analogous to symmetric double-well potentials in the continuum WKB case. In the context of Eq. (1.1), this means that $\mathbf{H} \| \hat{\mathbf{x}}$. The cases where $H_{y}$ or $H_{z}$ are also nonzero correspond to an asymmetric potential, and will be considered in a second paper.

Before describing the results of our analysis, however, let us digress to make two points. The first is the issue of degeneracy and its connection with symmetry in light of the von Neumann-Wigner theorem. When $\mathbf{H} \| \hat{\mathbf{x}}$, or $\mathbf{H} \| \hat{\mathbf{z}}, \mathcal{H}$ is invariant under a $180^{\circ}$ rotation about $\hat{\mathbf{x}}$ or $\hat{\mathbf{z}}$, so energies of levels that are odd and even under this operation can intersect. The quenchings for $\mathbf{H} \| \hat{\mathbf{x}}$ [Fig. [1(a)] can be understood as instances of this phenomenon [1](b). When $\mathbf{H}$ has both $\hat{\mathbf{x}}$ and $\hat{\mathbf{z}}$ components, however, $\mathcal{H}$ has no symmetry, and the level crossings [Fig. [1(b), (c)] are nontrivial instances of conical intersections or diabolical points [7.88]. Viewed in the larger $H_{x}-H_{z}$ plane, or the full three-dimensional space of magnetic fields $\mathbf{H}$, however, all points of degeneracy are diabolical.

The second point is that there are several other special features in the spectrum, which are evident from numerical analysis for several different $J$, and can also be seen in Fig. 1. First, the successive half-period shifts in the crossing fields as we go from (a) to (b) to (c) in Fig. 1 mean that the diabolical points form part of a centered rectangular lattice in the $H_{x}-H_{z}$ plane. The length of the rectangular unit cell along $H_{x}$ can be read off Eq. (1.2), while that along $H_{z}$ is given by [6, [9]

$$
\Delta H_{z}=\frac{\lambda^{1 / 2}}{J} H_{c},
$$

where $\lambda=k_{2} / k_{1}$. Second, at a diabolical point, we often find simultaneous degeneracy of more than one pair of levels to very high accuracy if not exactly. All these facts are captured by the DPI analysis. In fact, in the case $\mathbf{H} \| \hat{\mathbf{x}}$, all the available evidence to date exact diagonalization for small $J$, perturbation theory in $\lambda \equiv k_{2} / k_{1}$, numerics - indicates that the simultaneity of the degeneracy of many pairs of levels, as well as the values of the degeneracy fields, are exactly given by the leading semiclassical analysis, i.e., Eq. (1.2) [1](e). These facts point to the existence of a higher dynamical symmetry, but that is not yet established. Further, when higher order anisotropy terms are included in the Hamiltonian to obtain quantitative agreement with experimentally observed period [2], the numerical 
evidence indicates that although the simultaneous degeneracy of several pairs of levels and the perfect lattice of diabolical points are no longer exact properties, they continue to hold to rather good approximation [10].

The plan of the paper is as follows. In Sec. II we outline the DPI approach. The basic idea is that in the $J_{z}$ basis, Schrödinger's equation for Eq. (1.1) has the form of a recursion relation or difference equation, as opposed to a differential equation for a massive particle in a one dimensional potential $V(x)$. This difference equation can be solved in close analogy with the continuum WKB approximation. We will see that compared to previous DPI studies [11 [14] new types of turning points arise in the study of Eq. (1.1), because the recursion relation has five terms as opposed to three in the earlier studies. These turning points have no continuum analogue. Our present discussion will rely on physical arguments and correspondence with the continuum case. A more formal discussion is given in Ref. 15.

In Sec. [I] we develop an analogue of Herring's formula [16, 17] for problems leading to five term recursion relations. In the continuum case, for a symmetric double well potential $[V(-x)=V(x)]$, this formula expresses the splitting for the $n$th pair of levels in terms of the $x=0$ values of the wavefunction $\psi_{n}(x)$ and its derivative $\psi_{n}^{\prime}(x)$ for the $n$th state localized in one of the wells. In Sec. IV we will use the DPI method to find the analogous discrete wavefunction near the center of the potential, and use our Herring formula to obtain a completely general formula [See Eq. (4.38)] that applies to any eigenvalue problem in the form of a recursion relation. This latter formula is written in terms of an action integral that runs between turning points, in close analogy with the continuum case. This formula is inconvenient for practical applications, however, and so in subsection E of Sec. IV, we will transform it into another result [Eq. (4.39)] that only requires the evaluation of a small number of much simpler integrals. The second formula is also completely general, and has the advantage of making the $J \rightarrow \infty$ asymptotic structure of the splittings transparent. In Sec. $\mathrm{V}$, we will apply this lattermost formula to the $\mathrm{Fe}_{8}$ problem, and obtain the splitting for all pairs of levels. We will discuss the quenching points and several other aspects of our results, including comparison with numerics, work by other authors [18, 19, and the features that appear to be exact.

\section{SUMMARY OF THE DPI METHOD}

The starting point is to write Schrödinger's equation in the $J_{z}$ basis. Suppose $|\psi\rangle$ is an eigenstate of $\mathcal{H}$ with energy $E$. Then with $J_{z}|m\rangle=m|m\rangle,\langle m \mid \psi\rangle=C_{m},\langle m|\mathcal{H}| m\rangle=w_{m}$, and $\left\langle m|\mathcal{H}| m^{\prime}\right\rangle=t_{m, m^{\prime}}\left(m \neq m^{\prime}\right)$, we have

$$
\sum_{n=m-2}^{m+2} t_{m, n} C_{n}+w_{m} C_{m}=E C_{m}
$$

where the prime on the sum indicates that the term $n=m$ is to be omitted. The diagonal terms $\left(w_{m}\right)$ arise from the $J_{z}^{2}$ part of $\mathcal{H}$, the $t_{m, m \pm 1}$ terms from the $J_{x} H_{x}$ part, and the $t_{m, m \pm 2}$ terms from the $J_{x}^{2}$ part.

We can think of Eq. (2.1) as a tight binding model for an electron in a one-dimensional lattice with sites labelled by $m$, and slowly varying on-site energies $\left(w_{m}\right)$, nearest-neighbor $\left(t_{m, m \pm 1}\right)$, and next-nearest-neighbor $\left(t_{m, m \pm 2}\right)$ hopping terms. Since we can think of dynamics 
in this model in terms of wavepackets, it is clear that there is a generalization of the usual continuum quasiclassical or phase integral method to the lattice case. This is the DPI method.

Previous work with the DPI method [11]14 has been limited to the case where the recursion relation has only three terms. New features arise when five or more terms are considered. In particular, we encounter nonclassical turning points, i.e., turning points at $m$ values other than those at the limits of the classically allowed motion. It is these turning points that give rise to oscillatory tunnel splittings, so that this effect is absent in systems described by three-term recursion relations.

The general formalism of this method [13] and the extension to five terms is discussed at length elsewhere [15], so here we will only give a brief summary. The fundamental requirement for a quasiclassical approach to work is that $w_{m}$ and $t_{m, m \pm \alpha}(\alpha=1,2)$ vary slowly enough with $m$ that we can find smooth continuum approximants $w(m)$ and $t_{\alpha}(m)$, such that whenever $m$ is an eigenvalue of $J_{z}$, we have

$$
\begin{aligned}
w(m) & =w_{m}, \\
t_{\alpha}(m) & =\left(t_{m, m+\alpha}+t_{m, m-\alpha}\right) / 2, \quad \alpha=1,2 .
\end{aligned}
$$

We further demand that

$$
\frac{d w}{d m}=O\left(\frac{w(m)}{J}\right), \quad \frac{d t_{\alpha}}{d m}=O\left(\frac{t_{\alpha}(m)}{J}\right),
$$

with $m / J$ being treated as quantity of order 1. We will see that for Eq. (1.1), these conditions will hold in the semiclassical limit $J \gg 1$.

Given these conditions, the basic approximation, which readers will recognize from the continuum case, is to write the wavefunction as a linear combination of the quasiclassical forms

$$
C_{m} \sim \frac{1}{\sqrt{v(m)}} \exp \left(i \int^{m} q\left(m^{\prime}\right) d m^{\prime}\right),
$$

where $q(m)$ and $v(m)$ obey the equations

$$
\begin{aligned}
E & =w(m)+2 t_{1}(m) \cos q+2 t_{2}(m) \cos (2 q) \equiv \mathcal{H}_{\mathrm{sc}}(q, m), \\
v(m) & =\partial \mathcal{H}_{\mathrm{sc}} / \partial q=-2 \sin q(m)\left(t_{1}(m)+4 t_{2}(m) \cos q(m)\right) .
\end{aligned}
$$

Equations (2.6) and (2.7) are the lattice analogs of the eikonal and transport equations. Equation (2.5) represents the first two terms in an expansion of $\log C_{m}$ in powers of $1 / J$.

As in the continuum case, the approximate DPI wavefunction is invalid at turning points. These points arise whenever the velocity $v(m)$ vanishes for given energy $E$, for then the approximation (2.5) diverges. We see from Eq. (2.7) that $v(m)$ can vanish either because $\sin q=0$, i.e., $q=0$ or $q=\pi$, or because $q=q_{*} \equiv \cos ^{-1}\left(-t_{1} / 4 t_{2}\right)$. Substituting these values of $q$ in the eikonal equation, we see that a turning point is obtained whenever

$$
E=U_{0}(m), U_{\pi}(m), \text { or } U_{*}(m),
$$

where 


$$
\begin{aligned}
& U_{0}(m)=\mathcal{H}_{\mathrm{sc}}(0, m)=w(m)+2 t_{1}(m)+2 t_{2}(m), \\
& U_{\pi}(m)=\mathcal{H}_{\mathrm{sc}}(\pi, m)=w(m)-2 t_{1}(m)+2 t_{2}(m), \\
& U_{*}(m)=\mathcal{H}_{\mathrm{sc}}\left(q_{*}, m\right)=w(m)-2 t_{2}(m)-\frac{t_{1}^{2}(m)}{4 t_{2}(m)} .
\end{aligned}
$$

Note that at a turning point, both $m$ and $q$ are determined. If we denote the values of these quantities generically by $m_{c}$ and $q_{c}, m_{c}$ may be regarded as being fixed by Eq. (2.8), and $q_{c}$ by the corresponding condition $q_{c}=0, q_{c}=\pi$, or $q_{c}=q_{*}\left(m_{c}\right)$.

To understand the nature of these turning points, let us assume that $t_{1}<0$, and $t_{2}>0$. [This is the case for the Hamiltonian (1.1). We can always arrange for $t_{1}$ to be negative by means of the gauge transformation $C_{m} \rightarrow(-1)^{m} C_{m}$. Thus there is only one other case to be considered, namely, $t_{1}<0, t_{2}<0$. This is discussed in Ref. [15.] It then follows that $U_{\pi}>U_{0}$, and that

$$
U_{0}(m)-U_{*}(m)=\frac{1}{4 t_{2}(m)}\left(t_{1}(m)+4 t_{2}(m)\right)^{2} \geq 0 .
$$

Secondly, let us think of $\mathcal{H}(q, m)$ for fixed $m$ as an energy band curve. Then $U_{\pi}$ is always the upper band edge, while the lower band edge is either $U_{0}$ or $U_{*}$ according as whether $-t_{1} / 4 t_{2}$ is greater than or lesser than 1 . To deal with this possibility, it pays to introduce a dual labelling scheme for all three curves $U_{0}, U_{\pi}$, and $U_{*}$. We write $U_{\pi}(m) \equiv U_{+}(m)$, and

$$
\begin{array}{ll}
U_{0}(m)=U_{i}(m), U_{*}(m)=U_{-}(m), & \text { if } q_{*} \in(0, \pi), \\
U_{0}(m)=U_{-}(m), \quad U_{*}(m)=U_{f}(m), & \text { if } q_{*} \notin(0, \pi) .
\end{array}
$$

The subscripts + and - denote upper and lower band edges, while the subscripts $i$ and $f$ denote internal and forbidden respectively, since in the first case above, $U_{0}$ lies inside the energy band, while in the second case, $U_{*}$ lies outside. As examples of these curves for a symmetric recursion relation, we show those for $\mathrm{Fe}_{8}$ in Fig. 2. A magnified view of the lower left hand portion of this diagram is given in Fig. 月.

Turning points where $E=U_{+}$, or $E=U_{-}$when $U_{-}=U_{0}$, are analogous to those encountered in the continuum quasiclassical method, since the energy lies at a limit of the classically allowed range for the value of $m$ in question. Points where $E=U_{-}$when $U_{-}=U_{*}$ are physically analogous, but mathematically different since the value of $q_{c}$ is neither 0 nor $\pi$. Points where $E=U_{i}$ (see the energy $E_{1}$ in Fig. 2, e.g.) are novel in that the energy is inside the classically allowed range for $m_{c}$, but the mathematical form of the connection formulas is identical to the case $E=U_{-}=U_{0}$ since $q_{c}=0$. Most interesting are the turning points with $E=U_{f}$ (the point $m=-m_{1}$ in Fig. 3, for instance), since now the energy is outside the allowed range for $m=m_{c}$, and the value of $q_{c}$ is therefore necessarily complex. These points lie "under the barrier" and turn out to be the ones of importance for understanding oscillatory tunnel splittings.

The above discussion shows that the curves $U_{0}, U_{\pi}$, and $U_{*}$ collectively play the same role as the potential energy in the continuum quasiclassical method. We refer to them as critical curves. We have already noted that $U_{\pi}>U_{0} \geq U_{*}$. Let us suppose that the case of equality in Eq. (2.12) occurs at $m=m^{*}$. Clearly $t_{1}\left(m^{*}\right) / 4 t_{2}\left(m^{*}\right)=-1$, which is precisely the condition found above for the lower band edge to change from $q=0$ to $q=q_{*}$. Secondly, expanding $t_{1}$ and $t_{2}$ about $m *$, we see that $U_{0}$ and $U_{*}$ have a common tangent when they meet. 


\section{HERRING'S FORMULA FOR FIVE-TERM RECURSION RELATIONS}

The problem of computing tunnel splittings in a symmetric double well potential in the continuum case is greatly simplified by use of Herring's formula [16, 17]. An entirely analogous formula can be derived in the discrete case [20,14 (c) following the simplified treatment of Landau and Lifshitz [21].

We have already noted the importance of the critical curves. For low lying energy levels, in particular, the curve $U_{-}$is very much like the potential energy in the continuum case, and it is clear that we will have an entire series of approximate energy eigenstates with wavefunctions localized in any one of the two wells, in the vicinity of $\pm m_{0}$, the minima of $U_{-}(m)$. (See Fig. 2.) Let $C_{m}$ be the $n$th such wavefunction localized in the right hand well, normalized to unit total probability, and let it satisfy Schrödinger's equation (2.1) with an energy $E_{0}$ for all values of $m$ well to the right of the left well, including in particular the region around $m=0$. More precisely, we take $C_{m}$ to decay away from the right well in

both directions. Such a function could be obtained, e.g., as the energy eigenfunction of a modified problem in which the on-site energy is increased by a large positive amount for all $m<m_{a}$, where $-m_{0} \ll m_{a} \ll 0$, it being understood that $m_{a}$ is far away from all turning points for the energy concerned. However, this problem need not be solved explicitly, as the exact behavior of $C_{m}$ near $m=-m_{0}$ is never needed, and therefore need not be examined too closely.

Given such a function, Herring shows that the true symmetric and antisymmetric eigenfunctions, $s_{j}$ and $a_{j}$, with energies $E_{1}$ and $E_{2}$ respectively, are given very accurately by

$$
\begin{aligned}
& a_{m}=\frac{1}{\sqrt{2}}\left(C_{m}-C_{-m}\right), \\
& s_{m}=\frac{1}{\sqrt{2}}\left(C_{m}+C_{-m}\right) .
\end{aligned}
$$

The product $C_{m} C_{-m}$ is exponentially small everywhere, these functions are normalized to unit total probaility to exponentially high accuracy.

The Schrödinger equations obeyed by $C_{m}$ and $a_{m}$ are

$$
\begin{gathered}
\left(w_{m}-E_{0}\right) C_{m}+\sum_{n=m-2}^{m+2} t_{m, n} C_{n}=0, \\
\left(w_{m}-E_{1}\right) a_{m}+\sum_{n=m-2}^{m+2} t_{m, n} a_{n}=0 .
\end{gathered}
$$

Let us now define $m_{r}$ to be 1 if $J$ is integral, and $1 / 2$ when $J$ is half-integral. Multiplying Eq. (3.2) by $a_{m}$, Eq. (3.3) by $C_{m}$, and summing over $m$ from $m_{r}$ to $J$, we get

$$
\left(E_{1}-E_{0}\right) \sum_{m=m_{r}} C_{m} a_{m}+\Sigma_{1}-\Sigma_{2}=0,
$$

where

$$
\begin{aligned}
& \Sigma_{1}=\sum_{m=m_{r}}^{J} \sum_{n=m-2}^{m+2} a_{m} t_{m, n} C_{n}, \\
& \Sigma_{2}=\sum_{m=m_{r}}^{J} \sum_{n=m-2}^{m+2} C_{m} t_{m, n} a_{n} .
\end{aligned}
$$


To simplify Eq. (3.4), we first note that by Eq. (3.1)

$$
\sum_{m=m_{r}} C_{m} a_{m} \approx \frac{1}{\sqrt{2}} \sum_{m=m_{r}} C_{m}^{2} \approx \frac{1}{\sqrt{2}},
$$

since the product $C_{m} C_{-m}$ is everywhere exponentially small, and since $C_{m}^{2}$ is concentrated almost completely in the right well. Secondly, most of the terms in the sums $\Sigma_{1}$ and $\Sigma_{2}$ can be seen to be identical by shifting the summation indices in various terms suitably, and making use of the symmetry $t_{m, n}=t_{n, m}$. For example, the difference between the terms in $\Sigma_{1}$ with $n=m+2$, and those in $\Sigma_{2}$ with $n=m-2$ equals

$$
\begin{aligned}
\sum_{m=m_{r}}^{J}\left(a_{m} t_{m, m+2} C_{m+2}-C_{m} t_{m, m-2} a_{m-2}\right) & =\sum_{m=m_{r}}^{J} a_{m} t_{m, m+2} C_{m+2}-\sum_{m=m_{r}-2}^{J-2} C_{m+2} t_{m+2, m} a_{m} \\
& =-a_{m_{r}-1} t_{m_{r}-1, m_{r}+1} C_{m_{r}+1}-a_{m_{r}-2} t_{m_{r}-2, m_{r}} C_{m_{r}}
\end{aligned}
$$

where we have made use of the obvious facts that $t_{J, J+2}$ and $t_{J-1, J+1}$ are identically zero. The differences between the other terms in $\Sigma_{1}$ and $\Sigma_{2}$ can be similarly evaluated, and reduce to a small number of terms involving the product of an $a$ with a $C$, which can then be written entirely in terms of $C$ 's using Eq. (3.1). Finally, we can see that $E_{1}-E_{0}=E_{0}-E_{2}=$ $\pm \Delta / 2$, and the net result is that upto an irrelevant over all sign,

$$
\Delta=\left\{\begin{array}{lc}
2\left[t_{0,1} C_{0}\left(C_{1}-C_{-1}\right)+t_{0,2} C_{0}\left(C_{2}-C_{-2}\right)+t_{-1,1}\left(C_{1}^{2}-C_{-1}^{2}\right)\right], & \text { integer } J, \\
2 t_{-\frac{1}{2}, \frac{1}{2}}\left(C_{\frac{1}{2}}^{2}-C_{-\frac{1}{2}}^{2}\right)+4 t_{-\frac{3}{2}, \frac{1}{2}}\left(C_{\frac{1}{2}} C_{\frac{3}{2}}-C_{-\frac{1}{2}} C_{-\frac{3}{2}}\right), & \text { half-integer } J .
\end{array}\right.
$$

Herring gives a more careful justification of his formula by employing the Temple-Kato error bound on energy eigenvalues 22,23 . His argument can be adapted word for word to the present problem, and shows that the error in the splitting as calculated via Eq. (3.9) is exponentially smaller than the splitting itself, by a factor such as $e^{-c J}$ where $c>0$. As $J \rightarrow \infty$, therefore, Eq. (3.9) is asymptotically correct.

We remind readers that Eq. (3.9) is not limited to the ground state splitting.

\section{GENERAL FORMULA FOR TUNNEL SPLITTING IN TERMS OF ACTION INTEGRALS}

To apply Herring's formula (3.9) to the Hamiltonian (1.1), we will use the DPI approximation for the wavefunction. Actually, we will take $C_{m}$ in Eq. (3.9) to be localized in the left well. This can only change the answer by a sign, which is not of interest to us anyway.

\section{A. DPI form near potential well minimum}

Let us first take up the problem of finding the DPI approximation to $C_{m}$ in somewhat general terms. Step 1 is to find $C_{m}$ in the classically allowed region near $-m_{0}$, the minimum of $U_{-}(m)$. (See Fig. 3.) We assume, as will be seen to be true for Eq. (1.1), that in this region $U_{-}=U_{0}$. For energies close to $U_{-}\left(-m_{0}\right)$, and $m$ close to $-m_{0}$, the eikonal equation 
can only be satisfied if $q$ is close to zero. We can therefore expand $\mathcal{H}_{\mathrm{sc}}$ in powers of $m+m_{0}$ and $q$ :

$$
\mathcal{H}_{\mathrm{sc}}(q, m) \approx U_{-}\left(-m_{0}\right)+\frac{1}{2 M} q^{2}+\frac{1}{2} M \omega_{0}^{2}\left(m+m_{0}\right)^{2}+\cdots
$$

where

$$
\begin{aligned}
& M=-\left[2 t_{1}\left(-m_{0}\right)+8 t_{2}\left(-m_{0}\right)\right]^{-1}>0, \\
& \omega_{0}^{2}=-\left.2\left(t_{1}+4 t_{2}\right) \frac{\partial^{2} U_{-}}{\partial m^{2}}\right|_{m=-m_{0}} .
\end{aligned}
$$

Note that by virtue of Eq. (2.4), and its natural extension to second derivatives, $\omega_{0}$ is of order $1 / J$ relative to $t_{1}$ and $t_{2}$.

The allowed eigenvalues and eigenfunctions can now be written down very simply by noting that the eikonal equation is also the Hamilton-Jacobi equation with $q=\partial \Phi / \partial m, \Phi$ being the action. Thus the problem is identical to that of a harmonic oscillator. (Alternatively, we could arrive at the same result by approximating the original recurrence relation by a differential equation in the vicinity of $-m_{0}$.) For the $n$th state, therefore,

$$
E_{0}=U_{-}\left(-m_{0}\right)+\left(n+\frac{1}{2}\right) \omega_{0}
$$

and

$$
C_{m}=\left(2^{2 n}(n !)^{2} \pi \xi^{2}\right)^{-1 / 4} e^{-x^{2} / 2 \xi^{2}} H_{n}(x / \xi),
$$

where $x=m+m_{0}, H_{n}$ is the $n$th Hermite polynomial, and $\xi=\left(M \omega_{0}\right)^{-1 / 2}$. The wavefunction is already normalized, and the additional tails from the forbidden region only modify the normalization by an exponentially small amount.

It is apparent that the expansion (4.1) is invalid unless the point $-m_{0}$ is sufficiently far from the edge $m=-J$. Since the width of the wavefunction (4.5) is $\sqrt{n} \xi$, a necessary condition for the validity of our procedure is

$$
J-m_{0} \gg \sqrt{n} \xi .
$$

If this condition does not hold, then the recursion relation must be solved near the edge by a different method, which is tantamount to using the Holstein-Primakoff or Bogoliubov transformation. An example of the latter approach is given in Sec. IV of Ref. [24].

From the viewpoint of the DPI method, we have two turning points very close to $-m_{0}$, one to the left, and one to the right, since the condition $E=U_{-}(m)$ is then satisfied. The one to the left has been discussed above. Let us now consider the one to the right, and denote it by $-m_{t}$. We have

$$
-m_{t}+m_{0}=\left[\frac{2 n+1}{M \omega_{0}}\right]^{1 / 2} \sim(n J)^{1 / 2} .
$$

The neglected terms in Eq. (4.1), on the other hand, are of relative orders $q^{4},\left(m+m_{0}\right)^{3} / J^{3}$, and $\left(m+m_{0}\right) q^{2} / J$, and thus smaller than $n \omega_{0}$ for $x \ll\left(n J^{2}\right)^{1 / 3}$. Thus, provided $n \ll J$, the solution (4.5) holds well past $-m_{t}$, and can be matched directly onto the DPI solution under the barrier, without any need of connection formulas at $m=-m_{t}$ [25]. This argument is given at greater length in Sec. V of Ref. 24. 


\section{B. DPI form in ordinary forbidden region}

Step 2 is to consider the DPI solution for $m>-m_{t}$. Since we want this solution to decay as $m$ increases, we take it as

$$
C_{m}=\frac{B}{\sqrt{|v(m)|}} \exp \left(-\int_{-m_{t}}^{m} \kappa\left(m^{\prime}\right) d m^{\prime}\right),
$$

where $\kappa(m)=\operatorname{Im} q(m)>0$. This solution must be matched on to (4.5) to determine $B$. We can continue to use the harmonic oscillator approximation (4.1) to $\mathcal{H}_{\mathrm{sc}}$ for this purpose, and a simple calculation [26], which may in fact be traced back to Furry [27], leads to

$$
\begin{aligned}
B & =\left(\frac{\omega_{0} g_{n}}{2 \pi}\right)^{1 / 2} \\
g_{n} & =\frac{\sqrt{2 \pi}}{n !}\left(n+\frac{1}{2}\right)^{n+\frac{1}{2}} e^{-\left(n+\frac{1}{2}\right)} .
\end{aligned}
$$

The quantity $g_{n}$ is defined so that $g_{n} \rightarrow 1$ as $n \rightarrow \infty ; g_{0}=(\pi / e)^{1 / 2} \approx 1.075, g_{1} \approx 1.028$, $g_{2} \approx 1.017, \ldots$.

\section{DPI form in central region}

Step 3 is to find the wavefunction in the central region near $m=0$. This is already done if there are no turning points between $-m_{t}$ and $m=0$. For the Hamiltonian (1.1), it turns out that we encounter another turning point where $E=U_{f}(m)$ (the only possibility) at an intermediate point $m=-m_{1}$ (see Fig. 3). The solutions for $m<-m_{1}$ and $m>-m_{1}$ must therefore be related by a connection formula. To understand this turning point, we note that the eikonal equation (2.6) may be solved for $\cos q$ as

$$
\cos q(m)=\frac{-t_{1}(m) \pm\left[t_{1}^{2}(m)-4 t_{2}(m) f(m)\right]^{1 / 2}}{4 t_{2}(m)}
$$

where $f(m)=w(m)-2 t_{2}(m)-E$. Since $\cos q=-t_{1} / 4 t_{2}$ at $m=-m_{1}$, the discriminant in Eq. (4.11) must vanish, and we conclude that as we cross $-m_{1}, \cos q$ changes from real to complex, and $q$ changes from imaginary to complex. [Incidentally, it may be verified that the condition for vanishing discriminant, i.e.,

$$
t_{1}^{2}(m)=4 t_{2}(m)\left(w(m)-2 t_{2}(m)-E\right),
$$

is identical to $E=U_{*}(m)$.] Since the recursion relation is real, the solution $C_{m}$ must also be real for all $m$. A single DPI solution for $m>-m_{1}$ cannot meet this demand, and so we must take a linear combination of two DPI forms with complex conjugate $q$ 's. If we write these as

$$
q_{1,2}(m)=i \kappa(m) \pm \chi(m)
$$

with $\kappa$ and $\chi$ both real, then we must still have $\kappa>0$ in order that $C_{m}$ continue decaying, and we may also take $\chi>0$. Let us further write the solution (4.8) for $m<-m_{1}$ as 


$$
\begin{aligned}
C_{m} & =\frac{A}{2 \sqrt{|v(m)|}} \exp \left(-\int_{-m_{1}}^{m} \kappa\left(m^{\prime}\right) d m^{\prime}\right), \\
A & =2 B \exp \left(-\int_{-m_{t}}^{-m_{1}} \kappa\left(m^{\prime}\right) d m^{\prime}\right) .
\end{aligned}
$$

Then, as shown in Ref. [15], the DPI solution for $m>-m_{1}$ is given by

$$
C_{m}=\operatorname{Re} \frac{A}{\sqrt{s_{1}(m)}} \exp \left(i \int_{-m_{1}}^{m} q_{1}\left(m^{\prime}\right) d m^{\prime}\right),
$$

where $s_{1}(m)=-i v\left(q_{1}(m)\right)[28]$.

\section{Herring's formula with DPI approximation}

The solution (4.16) is ripe for substitution into the Herring formula (3.9). To do this, we first note that

$$
\begin{aligned}
\cosh \kappa \cos \chi & =-t_{1} / 4 t_{2}, \\
\sinh \kappa \sin \chi & =\left(4 t_{2} f-t_{1}^{2}\right)^{1 / 2} / 4 t_{2} .
\end{aligned}
$$

It then follows that

$$
s_{1}=8 t_{2}(m) \sinh \kappa(m) \sin \chi(m) \sin q_{1}(m) .
$$

We now substitute Eqs. (4.16)-(4.19) into Herring's formula, Eq. (3.9). In doing this, we may neglect the variation of quantities $t_{\alpha}(m), q(m)$, and $v(m)$ among the sites near the center of the lattice, since the number of sites involved is of order 1 , and so the variation leads to higher order corrections in powers of $1 / J$. To save writing, we denote quantities evaluated at $m=0$ by a bar: $q_{1}(0) \equiv \bar{q}_{1}, \kappa(0) \equiv \bar{\kappa}$, etc. We thus get

$$
C_{m}=\operatorname{Re} A_{2} \frac{e^{i\left(\Omega+m \bar{q}_{1}\right)}}{\sqrt{\sin \bar{q}_{1}}},
$$

where,

$$
\begin{aligned}
\Omega & =\int_{-m_{1}}^{0} q_{1}\left(m^{\prime}\right) d m^{\prime}, \\
A_{2} & =\left(8 \bar{t}_{2} \sinh \bar{\kappa} \sin \bar{\chi}\right)^{-1 / 2} A .
\end{aligned}
$$

The cases of integer and half-integer $J$ are best tackled separately. Doing the former first, we have

$$
\begin{aligned}
& C_{1}-C_{-1}=i A_{2}\left[e^{i \Omega} \sqrt{\sin \bar{q}_{1}}-\text { c.c. }\right] \\
& C_{1}+C_{-1}=A_{2}\left[e^{i \Omega} \sqrt{\frac{\cos ^{2} \bar{q}_{1}}{\sin \bar{q}_{1}}}+\text { c.c. }\right]
\end{aligned}
$$




$$
\begin{aligned}
C_{0}\left(C_{1}-C_{-1}\right) & =i \frac{A_{2}^{2}}{2}\left[\left(e^{2 i \Omega}-e^{-2 \operatorname{Im} \Omega} \sqrt{\frac{\sin \bar{q}_{1}^{*}}{\sin \bar{q}_{1}}}\right)-\text { c.c. }\right], \\
C_{2}-C_{-2} & =2 i A_{2}\left[e^{i \Omega} \cos \bar{q}_{1} \sqrt{\sin \bar{q}_{1}}-\text { c.c. }\right], \\
C_{0}\left(C_{2}-C_{-2}\right) & =i A_{2}^{2}\left[\left(e^{2 i \Omega} \cos \bar{q}_{1}-e^{-2 \operatorname{Im} \Omega} \cos \bar{q}_{1}^{*} \sqrt{\frac{\sin \bar{q}_{1}^{*}}{\sin \bar{q}_{1}}}\right)-\text { c.c. }\right], \\
C_{1}^{2}-C_{-1}^{2} & =i A_{2}^{2}\left[\cos \bar{q}_{1}\left(e^{2 i \Omega} \cos \bar{q}_{1}-e^{-2 \operatorname{Im} \Omega} \sqrt{\frac{\sin \bar{q}_{1}^{*}}{\sin \bar{q}_{1}}}\right)-\text { c.c. }\right] .
\end{aligned}
$$

Substituting these and the formula $\bar{t}_{1}=-4 \bar{t}_{2} \cosh \bar{\kappa} \cos \bar{\chi}$ into Eq. (3.9), we get

$$
\begin{aligned}
& \Delta=-8 A_{2}^{2} \bar{t}_{2} \operatorname{Im}\left(e^{2 i \Omega} \Theta-e^{-2 \operatorname{Im} \Omega} \sqrt{\frac{\sin \bar{q}_{1}^{*}}{\sin \bar{q}_{1}}} \operatorname{Re} \Theta\right) \\
& \Theta=\cos \bar{q}_{1}-\cosh \bar{\kappa} \cos \bar{\chi} .
\end{aligned}
$$

But, it follows from Eq. (4.13) that

$$
\cos \bar{q}_{1}=\cosh \bar{\kappa} \cos \bar{\chi}-i \sinh \bar{\kappa} \sin \bar{\chi},
$$

so the second term in Eq. (4.29) vanishes altogether, and

$$
\begin{aligned}
\Delta & =4 A_{2}^{2} \bar{t}_{2} \sinh \bar{\kappa} \sin \bar{\chi}\left(e^{2 i \Omega}+e^{-2 i \Omega^{*}}\right) \\
& =\frac{1}{2} A^{2}\left(e^{2 i \Omega}+e^{-2 i \Omega^{*}}\right)
\end{aligned}
$$

where we have used Eq. (4.22) in the last step.

For half-integer $J$, we get

$$
\begin{gathered}
C_{ \pm 1 / 2}^{2}=\frac{A_{2}^{2}}{4}\left[\left(\frac{e^{2 i \Omega}}{\sin \bar{q}_{1}} e^{ \pm i \bar{q}_{1}}+\frac{e^{-2 \operatorname{Im} \Omega}}{\left|\sin \bar{q}_{1}\right|}\right)+\text { c.c. }\right] \\
C_{ \pm 1 / 2} C_{ \pm 3 / 2}=\frac{A_{2}^{2}}{4}\left[\left(\frac{e^{2 i \Omega}}{\sin \bar{q}_{1}} e^{ \pm 2 i \bar{q}_{1}}+\frac{e^{-2 \operatorname{Im} \Omega}}{\left|\sin \bar{q}_{1}\right|} e^{\mp i \bar{q}_{1}}\right)+\text { c.c. }\right] .
\end{gathered}
$$

Thus,

$$
\begin{aligned}
C_{1 / 2}^{2}-C_{-1 / 2}^{2} & =\frac{i}{2} A_{2}^{2}\left(e^{2 i \Omega}-\text { c.c. }\right) \\
C_{1 / 2} C_{3 / 2}-C_{-1 / 2} C_{-3 / 2} & =i A_{2}^{2}\left(\cos \bar{q}_{1} e^{2 i \Omega}-\text { c.c. }\right),
\end{aligned}
$$

and

$$
\begin{aligned}
\Delta & =i A_{2}^{2}\left[\left(\bar{t}_{1}+4 \bar{t}_{2}\right) e^{2 i \Omega}-\text { c.c. }\right] \\
& =4 A_{2}^{2} \bar{t}_{2} \sinh \bar{\kappa} \sin \bar{\chi}\left(e^{2 i \Omega}+e^{-2 i \Omega^{*}}\right),
\end{aligned}
$$

which leads, once again, to Eq. (4.32).

Collecting Eqs. (4.9), (4.15), (4.21) and (4.32), and making use of the symmetry of the problem, we may write the tunnel splitting for both integer and half-integer $J$ as 


$$
\Delta=\frac{\omega_{0} g_{n}}{2 \pi}\left[\exp \left(i \int_{-m_{t}}^{m_{t}} q\left(m^{\prime}\right) d m^{\prime}\right)+\text { c.c. }\right]
$$

Here $q\left(m^{\prime}\right)$ is chosen to have a positive real part $\chi$ in the first term. We note once again that this result applies to higher pairs of excited states, and not just the ground pair. The essential dependence on $n$, the excitation number, enters through the $n$ dependence of $m_{t}$, the turning point.

The similarity of Eq. (4.38) to the final result in Ref. [21] is striking [29], and one can ask whether one should not have anticipated it right away. For the ground state pair, the instanton approach [1](a) makes it very easy to understand the presence of two complex conjugate tunneling actions, and the fact that they should be superposed, but does not give the prefactor. The action integrals in the instanton approach, however, run not from turning point to turning point but from one minimum of the energy to the other. Further, properly justifiying the prefactor using instantons has proven very difficult [30,31]. Purely as a recipe for calculations, however, a hybrid approach, in which one adds the tunneling actions from all equivalent instantons, and uses the DPI approach to determine the form of the prefactor, would appear to be valid for all problems. Thus, we strongly suspect that Eq. (4.38) is correct even when the recursion relation has seven or more terms.

\section{E. Extraction of singular parts of action integrals}

While the formula (4.38) is very general, it has the disadvantage that the action integral runs between turning points. The integrand is therefore close to a singularity, and for low lying states, this gives rise to terms in the action that depend on $\ln J$. Hence the formula does not reveal the asymptotic behavior as a function of $J$ in a transparent way.

In this subsection, we will show that we can write the splitting very simply in a way that does not suffer from the above drawback. The final result is

$$
\Delta_{n}=\frac{1}{n !} \sqrt{\frac{8}{\pi}} \omega_{0} F^{n+\frac{1}{2}} e^{-\Gamma_{0}} \cos \Lambda_{n},
$$

where,

$$
\begin{aligned}
\Gamma_{0} & =2 \int_{-m_{0}}^{0} \kappa_{0}(m) d m \\
\Lambda_{n} & =2 \int_{-m_{1}}^{0}\left(\chi_{0}+\left(n+\frac{1}{2}\right) \omega_{0} \chi_{0}^{\prime}\right) d m \\
F & =2 M \omega_{0}\left(m-m_{1}\right)^{2} \exp \left(-2\left(Q_{1}+\omega_{0} \int_{-m_{1}}^{0} \kappa_{0}^{\prime} d m\right)\right), \\
Q_{1} & =\int_{-m_{0}}^{-m_{1}}\left(\frac{\omega_{0} B_{0}^{\prime}}{\sqrt{B_{0}^{2}-1}}+\frac{1}{m+m_{0}}\right) d m .
\end{aligned}
$$

In Eqs. (4.40 4.43), the irregular turning points $\pm m_{1}$ may be evaluated by setting $E=$ $U_{-}\left( \pm m_{0}\right)$, and it should be recalled that $\pm m_{0}$ are the minima of $U_{-}(m)$. Further, 


$$
\begin{aligned}
& \kappa_{0}=\kappa(m, \epsilon=0) ; \quad \kappa_{0}^{\prime}=\left.\frac{\partial \kappa(m, \epsilon)}{\partial \epsilon}\right|_{\epsilon=0}, \\
& \chi_{0}=\chi(m, \epsilon=0) ; \quad \chi_{0}^{\prime}=\left.\frac{\partial \chi(m, \epsilon)}{\partial \epsilon}\right|_{\epsilon=0}, \\
& B_{0}=\cos q(m, \epsilon=0) ; \quad B_{0}^{\prime}=\left.\frac{\partial \cos q(m, \epsilon)}{\partial \epsilon}\right|_{\epsilon=0}
\end{aligned}
$$

with

$$
\epsilon \equiv E-U_{-}\left(-m_{0}\right)
$$

The problem of finding the low level splittings is thus reduced to the evaluation of a handful of integrals. The proliferation of notation masks the actual simplicty of these formulas.

To derive these results, we follow a procedure similar to that used for the continuum case in Ref. [26]. We begin by defining

$$
\Phi(\epsilon)=-i \int_{-m_{t}(\epsilon)}^{0} q(m, \epsilon) d m
$$

where the energy dependence is made explicit. The splitting for the $n$th pair of states is then given by

$$
\Delta_{n}=\frac{\omega_{0} g_{n}}{2 \pi}\left(e^{-2 \Phi\left(\epsilon_{n}\right)}+\text { c.c. }\right),
$$

with $\epsilon_{n}=\left(n+\frac{1}{2}\right) \omega_{0}$. Writing $x=m+m_{0}$ as in Eq. (4.5), the integrand in $\Phi$ behaves as $\left(x^{2}-x_{t}^{2}\right)^{1 / 2}$ near the lower limit, with $x_{t}=-m_{t}+m_{0} \sim \epsilon^{1 / 2}$. Thus there is a singular part in $\Phi$ of the form $\epsilon \ln \epsilon$, which it is our goal to extract. To this end, we differentiate Eq. (4.48) to get

$$
\Phi^{\prime}(\epsilon)=\frac{d \Phi}{d \epsilon}=-i \int_{-m_{t}(\epsilon)}^{0} \frac{\partial q}{\partial \epsilon} d m
$$

Note that the term arising from differentiating the lower limit vanishes, nor is there any explicit contribution from the singular behavior $q \sim\left(m+m_{c}\right)^{1 / 2}$ for $m$ near $-m_{c}$.

Next, let us divide $\Phi^{\prime}(\epsilon)$ into two integrals, $\Phi_{1}^{\prime}$, in which the limits of integration are $-m_{t}$ and $-m_{1}$, and $\Phi_{2}^{\prime}$, which runs from $-m_{1}$ to 0 . Defining

$$
B_{\epsilon}(m)=\cos (q(m, \epsilon))
$$

we have

$$
\Phi_{1}^{\prime}(\epsilon)=\int_{-m_{t}(\epsilon)}^{0} \frac{B_{\epsilon}^{\prime}}{\sqrt{B_{\epsilon}^{2}(m)-1}} d m,
$$

where $B_{\epsilon}^{\prime}=\partial B_{\epsilon} / \partial \epsilon$. It follows from Eq. (4.1) that near $m=-m_{t}$,

$$
B_{\epsilon} \approx 1+\left(\frac{1}{2} M \omega^{2} x^{2}-\epsilon\right) M+\cdots,
$$


so the integrand in Eq. (4.52) behaves as $-1 / \omega_{0}\left(x^{2}-x_{t}^{2}\right)^{1 / 2}$. If we add and subtract the integral of this expression, we obtain

$$
\Phi_{1}^{\prime}(\epsilon)=-\frac{1}{\omega_{0}} \int_{x_{t}}^{x_{1}} \frac{d x}{\sqrt{x^{2}-x_{t}^{2}}}+\int_{x_{t}}^{x_{1}}\left[\frac{B_{\epsilon}^{\prime}}{\sqrt{B_{\epsilon}^{2}(m)-1}}+\frac{1}{\omega_{0} \sqrt{x^{2}-x_{t}^{2}}}\right] d x,
$$

where $x_{1}=m_{0}-m_{1}$. The first integral can be evaluated exactly. In the second integral we can put $\epsilon=0$ both in the limits and in the integrand, since we are not interested in terms of $O(\epsilon)$. Ignoring terms of this order throughout, and making use of the relation

$$
x_{t}^{2}=2 \epsilon / M \omega_{0}^{2}
$$

we obtain

$$
\Phi_{1}^{\prime}(\epsilon)=\frac{1}{2 \omega_{0}}\left[\ln \frac{\epsilon}{2 M \omega_{0}\left(m_{0}-m_{1}\right)^{2}}+2 Q_{1}\right],
$$

where $Q_{1}$ is given by Eq. (4.43). Also, we can evaluate $m_{1}$ at $\epsilon=0$.

The remaining contribution to $\Phi^{\prime}(\epsilon), \Phi_{2}^{\prime}(\epsilon)$, can be evaluated simply by putting $\epsilon=0$, since the neglected part is $O(\epsilon)$. Recalling the definitions (4.44) and (4.45), we have

$$
\Phi_{2}^{\prime}(\epsilon) \approx \int_{-m_{1}}^{0}\left(\kappa_{0}^{\prime}-i \chi_{0}^{\prime}\right) d m
$$

We now integrate the expression for $\Phi^{\prime}(\epsilon)$ and obtain $\Phi$. It is useful to separate the real and imaginary parts of the answer at this stage. For the real part, we get

$$
\Gamma=2 \operatorname{Re} \Phi=\Gamma_{0}+\frac{\epsilon}{\omega_{0}}\left[2 Q_{1}-1+\ln \frac{\epsilon}{2 M \omega_{0}\left(m_{0}-m_{1}\right)^{2}}+2 \omega_{0} \int_{-m_{1}}^{0} \kappa_{0}^{\prime} d m\right],
$$

with $\Gamma_{0}$ given by Eq. (4.40), while for the imaginary part, $\Lambda_{n} \equiv-2 \operatorname{Im} \Phi$, we get Eq. (4.41).

Substituting Eqs. (4.58)-(4.41), and the definition (4.10) of $g_{n}$ in the formula (4.49) for $\Delta_{n}$, and recalling that $\epsilon_{n}=\left(n+\frac{1}{2}\right) \omega_{0}$, we finally obtain the answer quoted at the start, Eq. (4.39).

\section{APPLiCATion TO THE Fe 8 PROBLEM}

We now apply our general formulas to the specific problem of $\mathrm{Fe}_{8}$, as described by the Hamiltonian (1.1). The various matrix elements of this Hamiltonian are given by

$$
\begin{aligned}
w_{m} & =\frac{1}{2}\left(k_{1}+k_{2}\right)\left[J(J+1)-m^{2}\right], \\
t_{m, m+1} & =-\frac{1}{2} g \mu_{B} H_{x}[J(J+1)-m(m+1)]^{1 / 2}, \\
t_{m, m+2} & =\frac{1}{4}\left(k_{1}-k_{2}\right)[[J(J+1)-m(m+1)][J(J+1)-(m+1)(m+2)]]^{1 / 2} .
\end{aligned}
$$

We must now replace these by continuous functions $w(m), t_{1}(m)$, and $t_{2}(m)$. Since our formalism requires knowing the first two terms in the action in an expansion in powers of 
$1 / J$, it follows that we need only determine the functions $w(m)$ etc. to the same order. Furthermore, this determination need not be made in the form of a power series, and any functional representation that gives the first two terms correctly will be adequate. The most convenient way to do this is to replace the combination $J(J+1)$ in the above expressions by $\bar{J}^{2}$, where

$$
\bar{J}=J+\frac{1}{2} .
$$

The evaluation of the integrals (4.50)-(4.43) is then lengthy, but straightforward. We will present and discuss the final results first, and give the details of the analysis later.

\section{A. Tunnel splittings for $\mathrm{Fe}_{8}$}

The final result for the splitting of the $n$th pair of levels is

$$
\Delta_{n}=\frac{1}{n !} \sqrt{\frac{8}{\pi}} \omega_{0} F^{n+\frac{1}{2}} e^{-\Gamma_{0}} \cos \Lambda_{n},
$$

where,

$$
\begin{aligned}
\omega_{0} & =2 J\left[k_{1} k_{2}\left(1-h_{x 0}^{2}\right)\right]^{1 / 2}, \\
F & =8 J \frac{\lambda^{1 / 2}\left(1-h_{x}^{2}\right)^{3 / 2}}{1-\lambda-h_{x}^{2}}, \\
\Gamma_{0} & =\bar{J}\left[\ln \left(\frac{\sqrt{1-h_{x}^{2}}+\sqrt{\lambda}}{\sqrt{1-h_{x}^{2}}-\sqrt{\lambda}}\right)-\frac{h_{x}}{\sqrt{1-\lambda}} \ln \left(\frac{\sqrt{\left(1-h_{x}^{2}\right)(1-\lambda)}+h_{x} \sqrt{\lambda}}{\sqrt{\left(1-h_{x}^{2}\right)(1-\lambda)}-h_{x} \sqrt{\lambda}}\right)\right], \\
\Lambda_{n} & =\max \left\{0, \pi J\left(1-\frac{H_{x}}{\sqrt{1-\lambda} H_{c}}\right)-n \pi\right\} .
\end{aligned}
$$

In Eqs. (5.6 5.9), $\lambda=k_{2} / k_{1}$, and

$$
h_{x}=\frac{J H_{x}}{\bar{J} H_{c}}, \quad h_{x 0}=\frac{H_{x}}{H_{c}} .
$$

Recall that $H_{c}=2 k_{1} J / g \mu_{B}$.

Let us now turn to the discussion of these results. The first point concerns the fields where the $n$th tunnel splitting vanishes. Taking account of the fact that $\Lambda_{n}$ is necessarily positive as indicated by Eq. (5.9), we see that this happens whenever [9.] (d,e)

$$
\frac{H_{x}}{H_{c}}=\frac{\sqrt{1-\lambda}}{J}\left[J-\ell-\frac{1}{2}\right],
$$

with $\ell=n, n+1, \ldots, 2 J-n-1$, yielding $2(J-n)$ quenching points in all for $\Delta_{n}$. When $n=0$, these are the results quoted in Sec. 固.

In Fig. 1 we compare Eq. (5.5) with the numerically evaluated splittings for the first three pairs of levels. Within our numerical precision, we always find the zeros of $\Delta_{n}$ to agree with Eq. (5.11). Note, however, that for other values of $H_{c}$, the discrepancy between the 
numerics and Eq. (5.5) is well outside our numerical error, so that Eq. (5.5) is not exact, even though as an asymtotic estimate of the splitting it is rather good. This means that the leading semiclassical approximation does not give the eigenvalues themselves exactly, and only the quenching points appear to be so reproduced. The second point to note is that for $n=1$ (the pair of first excited states in each well), the highest field quenching point is lost, for $n=2$, the highest two points are lost, and so on, exactly as indicated by Eq. (5.11).

Next, let us compare our answers with previous work. Let us consider the Gamow factor $\Gamma_{0}$ first. Except for the replacement of $J$ by $\bar{J}$ and $h_{x 0}$ by $h_{x}$, this is precisely the action in Eq. (3.10) of Ref. [1] (c). This agreement is unsurprising, because if we write $\Delta_{n}$ in the form of a prefactor $c_{1}$ times a Gamow factor $\exp \left(-J c_{2}\right)$ where $c_{2}=O(1)$, then the $J \rightarrow \bar{J}$, and $h_{x 0} \rightarrow h_{x}$ corrections in Eq. (5.8) represent terms that should be included in the prefactor $c_{1}$, which we did not seek to find in Ref. [1](c). The detailed form of the prefactor is perhaps more interesting. Up to multiplicative terms of order $J^{0}$, our answer for $\Delta_{n}$ agrees precisely with that in Ref. 18, 19. We do not understand, however, how these papers have succeeded in sidestepping the difficulties in the path integral treatment that were noted by Enz and Schilling [30], and by Belinicher, Providencia, and da Providencia [31]. In Ref. [18], for instance, the problem is treated by writing the spin coherent state expectation value of the Hamiltonian (1.1) in spherical polar coordinates, and integrating out $\cos \theta$ (the $J_{z}$ projection) exactly, and then addressing the resulting effective Lagrangian for the $\phi$ coordinate exactly as for a massive particle in one dimension. In performing the integration over $\theta$, however, it is not clear to us why $S^{2}$ is replaced by $S(S+1)$ in the scalar potential $V(\phi)$ [see Eq. (12) there], but not in the vector potential $\Theta(\phi)$.

A related point, which is relatively minor, but has scope for creating confusion, is that if the Gamow factor is written as $\exp \left(-J c_{2}\right)$ with $c_{2}=O(1)$, then it is safest to write the $J$ dependence of the prefactor as $\omega_{0} J^{n+1 / 2}$, since $\omega_{0}$ depends on parameters such as $k_{1}$ and $k_{2}$, whose scaling with $J$ is a matter of choice, at least as far as model Hamiltonians are concerned.

One further check is obtained by considering the limiting case $H_{x}=0$, answers for which are known. [See, e.g., Eq. (16) of [30], Eq. (48) of [31], or [24].] Transcribing Eqs. (4.30) and (4.31) from [24] in terms of the present parameters, we get

$$
\begin{aligned}
\Delta_{n} & =\frac{1}{n !} F_{0}^{n} \Delta_{0} \\
F_{0} & =8 J \frac{\sqrt{\lambda}}{1-\lambda} \\
\Delta_{0} & =8 \omega_{00}\left(\frac{J}{\pi}\right)^{1 / 2} \frac{\lambda^{1 / 4}}{1+\sqrt{\lambda}}\left(\frac{1-\sqrt{\lambda}}{1+\sqrt{\lambda}}\right)^{J},
\end{aligned}
$$

with $\omega_{00}=2 J\left(k_{1} k_{2}\right)^{1 / 2}$. It follows from Eqs. (5.6 5.9) that as $H_{x} \rightarrow 0, \omega_{0} \rightarrow \omega_{00}, F \rightarrow F_{0}$ [see Eq. (5.7)], $\cos \Lambda_{n} \rightarrow \pm 1$, and

$$
\Gamma_{0} \rightarrow\left(J+\frac{1}{2}\right) \ln \frac{1+\sqrt{\lambda}}{1-\sqrt{\lambda}} .
$$

It is then easy to see that our present answers for $\Delta_{n}$ go over precisely into Eqs. (5.12)-(5.14). 


\section{B. Evaluation of Action Integrals}

To carry out the evaluation of Eqs. (4.50)-(4.43), it is convenient to measure energies

(including $\omega_{0}$ ) in units of $k_{1} \bar{J}^{2}$, and introduce the scaled variable $\mu=m / \bar{J}$. In terms of these variables, we have

$$
\begin{aligned}
& w(m)=(1+\lambda)\left(1-\mu^{2}\right) / 2, \\
& t_{1}(m)=-h_{x}\left(1-\mu^{2}\right)^{1 / 2}, \\
& t_{2}(m)=(1-\lambda)\left(1-\mu^{2}\right) / 4 .
\end{aligned}
$$

The turning points $\mu_{0}=m_{0} / \bar{J}$, and $\mu_{1}=m_{1} / \bar{J}$ (for $\epsilon=0$ ) are given by

$$
\begin{aligned}
& \mu_{0}=\left(1-h_{x}^{2}\right)^{1 / 2} \\
& \mu_{1}=\left[\left(1-\lambda-h_{x}^{2}\right) /(1-\lambda)\right]^{1 / 2} .
\end{aligned}
$$

It is most convenient to express everything in terms of $\mu_{0}$ and $\mu_{1}$, so we give inverse formulas as well:

$$
\begin{array}{r}
h_{x}=\left(1-\mu_{0}^{2}\right)^{1 / 2}, \\
\lambda=\left(\mu_{0}^{2}-\mu_{1}^{2}\right) /\left(1-\mu_{1}^{2}\right) .
\end{array}
$$

The mass and the small oscillation frequency are given by

$$
\begin{array}{r}
M=\frac{1}{2 \lambda h_{x}^{2}}=\frac{1}{2} \frac{1-\mu_{1}^{2}}{\left(1-\mu_{0}^{2}\right)\left(\mu_{0}^{2}-\mu_{1}^{2}\right)}, \\
\omega_{0}=2\left[\lambda\left(1-h_{x}^{2}\right)\right]^{1 / 2} / \bar{J}=\frac{2 \mu_{0}}{\bar{J}}\left(\frac{\mu_{0}^{2}-\mu_{1}^{2}}{1-\mu_{1}^{2}}\right)^{1 / 2} .
\end{array}
$$

To evaluate the integrals, we need expressions for $\kappa_{0}, \chi_{0}, \kappa_{0}^{\prime}$, etc. in the ranges $\mu_{1}<$ $\mu<\mu_{0}$, and $0<\mu<\mu_{1}$. The requisite calculations are straightforward so we give the main results only. First, in the range $\mu_{1}<\mu<\mu_{0}$, we get

$$
\begin{aligned}
& B_{0}=\cosh \kappa_{0}=\frac{1-\mu_{1}^{2}-\left[\left(\mu_{0}^{2}-\mu_{1}^{2}\right)\left(\mu^{2}-\mu_{1}^{2}\right)\right]^{1 / 2}}{\left[\left(1-\mu_{0}^{2}\right)\left(1-\mu^{2}\right)\right]^{1 / 2}} \\
& \sqrt{B_{0}^{2}-1}=\sinh \kappa_{0}=\frac{\left(\sqrt{\mu_{0}^{2}-\mu_{1}^{2}}-\sqrt{\mu^{2}-\mu_{1}^{2}}\right) \sqrt{1-\mu_{1}^{2}}}{\left[\left(1-\mu_{0}^{2}\right)\left(1-\mu^{2}\right)\right]^{1 / 2}} \\
& B_{0}^{\prime}=-\frac{1}{2} \frac{1-\mu_{1}^{2}}{\left[\left(1-\mu_{0}^{2}\right)\left(1-\mu^{2}\right)\left(\mu_{0}^{2}-\mu_{1}^{2}\right)\left(\mu^{2}-\mu_{1}^{2}\right)\right]^{1 / 2}} \\
& \frac{\omega_{0} B_{0}^{\prime}}{\sqrt{B_{0}^{2}-1}}=-\frac{\mu_{0}}{\bar{J}} \frac{1}{\sqrt{\mu^{2}-\mu_{1}^{2}}\left(\sqrt{\mu_{0}^{2}-\mu_{1}^{2}}-\sqrt{\mu^{2}-\mu_{1}^{2}}\right)}
\end{aligned}
$$

Next, in the range, $0<\mu<\mu_{1}$, we first put $\epsilon=0$ in Eqs. (4.17) and (4.18), and solve, to obtain 


$$
\begin{aligned}
\cosh \kappa_{0} & =\left[\left(1-\mu_{1}^{2}\right) /\left(1-\mu_{0}^{2}\right)\right]^{1 / 2}, \\
\cos \chi_{0} & =\left[\left(1-\mu_{1}^{2}\right) /\left(1-\mu^{2}\right)\right]^{1 / 2} . \\
\sin \chi_{0} & =\left[\left(\mu_{1}^{2}-\mu^{2}\right) /\left(1-\mu^{2}\right)\right]^{1 / 2}
\end{aligned}
$$

We then differentiate Eqs.4.17) and (4.18), and set $\epsilon=0$ to obtain the equations

$$
\begin{aligned}
& \sinh \kappa_{0} \cos \chi_{0} \kappa_{0}^{\prime}-\cosh \kappa_{0} \sin \chi_{0} \chi_{0}^{\prime}=0, \\
& \cosh \kappa_{0} \sin \chi_{0} \kappa_{0}^{\prime}+\sinh \kappa_{0} \cos \chi_{0} \chi_{0}^{\prime}=-1 / 8 t_{2} \sinh \kappa_{0} \sin \chi_{0} .
\end{aligned}
$$

Solving these, we obtain

$$
\left(\begin{array}{c}
\kappa_{0}^{\prime} \\
\chi_{0}^{\prime}
\end{array}\right)=-\frac{\left(1-\mu_{1}^{2}\right)^{1 / 2}}{2\left(\mu_{0}^{2}-\mu^{2}\right)}\left(\begin{array}{c}
\left(\mu_{0}^{2}-\mu_{1}^{2}\right)^{-1 / 2} \\
\left(\mu_{1}^{2}-\mu^{2}\right)^{-1 / 2}
\end{array}\right) .
$$

The first integral that we wish to evaluate is $\Gamma_{0}$, which will give us the dominant WKB or Gamow factor in the tunnel splitting. We divide the integral into two parts by breaking the integration range at $m_{1}$. From the right-hand part, an integration by parts gives

$$
\begin{aligned}
\Gamma_{01} & =2 \bar{J} \int_{\mu_{1}}^{\mu_{0}} \kappa_{0} d \mu \\
& =2 \bar{J}\left[\left.\kappa_{0}(\mu) \mu\right|_{\mu_{1}} ^{\mu_{0}}-\int_{\mu_{1}}^{\mu_{0}} \frac{\mu}{\sinh \kappa_{0}} \frac{d B_{0}(\mu)}{d \mu} d \mu\right],
\end{aligned}
$$

while from the left-hand part we get

$$
\Gamma_{02}=2 \bar{J} \int_{0}^{\mu_{1}} \kappa_{0} d \mu=2 \bar{J} \kappa_{0}\left(\mu_{1}\right) \mu_{1},
$$

as $\kappa_{0}$ is a constant in this range. Since $\kappa_{0}\left(\mu_{0}\right)=0, \Gamma_{02}$ cancels the first term in Eq. (5.35), leaving us only with the second for $\Gamma_{0}$. Using Eqs. (5.25) and (5.26), we find

$$
\Gamma_{0}=2 \bar{J}\left(1-\mu_{1}^{2}\right)^{1 / 2} \int_{\mu_{1}}^{\mu_{0}} \frac{d \mu}{\left(1-\mu^{2}\right)\left(\mu^{2}-\mu_{1}^{2}\right)^{1 / 2}} .
$$

The integration is now elementary, and the result, expressed back in terms of $\lambda$ and $h_{x}$ is Eq. (5.8).

The second integral to be evaluated is $\Lambda_{n}$. For the first term in Eq. (4.41), we integrate by parts, and use Eqs. (5.30) and (5.31):

$$
\begin{aligned}
2 \bar{J} \int_{0}^{\mu_{1}} \chi_{0}(\mu) d \mu & =2 \bar{J} \int_{0}^{\mu_{1}} \frac{\mu}{\sin \chi_{0}} \frac{d}{d \mu} \cos \chi_{0} d \mu \\
& =2 \bar{J} \int_{0}^{\mu_{1}} \frac{\mu^{2}}{\left(1-\mu^{2}\right)\left(\mu_{1}^{2}-\mu^{2}\right)^{1 / 2}} d \mu \\
& =\pi \bar{J}\left[1-\left(1-\mu_{1}^{2}\right)^{1 / 2}\right] .
\end{aligned}
$$

For the second term in Eq. (4.41), we have, with $\epsilon=\left(n+\frac{1}{2}\right) \omega_{0}$, and Eqs. (5.24) and (5.34),

$$
\begin{aligned}
2 \epsilon \bar{J} \int_{0}^{\mu_{1}} \kappa_{0}^{\prime} d \mu & =-(2 n+1) \mu_{0}\left(\mu_{0}^{2}-\mu_{1}^{2}\right)^{1 / 2} \int_{0}^{\mu_{1}} \frac{d \mu}{\left(\mu_{0}^{2}-\mu^{2}\right)\left(\mu_{1}^{2}-\mu^{2}\right)^{1 / 2}} \\
& =-\left(n+\frac{1}{2}\right) \pi .
\end{aligned}
$$


Adding together the parts, and rewriting the result in terms of $H_{x}$ and $\lambda$, we get Eq. (5.9). The restriction that $\Lambda_{n}$ be positive follows from the fact that we chose $q(m)$ to have a positive real part in Eq. (4.38). Thus $\Lambda$ is necessarily positive as defined in Eq. (4.41). If $H_{x}$ is so large as to yield a negative value for the function of $H_{x}$ that results after doing the integral, that means that in fact there are no irregular turning points in the problem. Both terms in Eq. (4.38) are then equal, and the formula reduces to the expected one when there are only regular turning points.

Note that unlike Eq. (5.8), what appears in Eq. (5.9) is the ratio $H_{x} / H_{c}$, i.e., $h_{x 0}$, not $h_{x}$. This fact is important for the location of the diabolical points.

The third integral we need is that of $\kappa_{0}^{\prime}$ from 0 to $\mu_{1}$. Using Eqs. (5.24) and (5.34), we get

$$
2 \omega_{0} \bar{J} \int_{0}^{\mu_{1}} \kappa_{0}^{\prime} d \mu=-2 \mu_{0} \int_{0}^{\mu_{1}} \frac{d \mu}{\mu_{0}^{2}-\mu^{2}}=\ln \frac{\mu_{0}-\mu_{1}}{\mu_{0}+\mu_{1}} .
$$

The fourth and last integral needed is $Q_{1}$. Substituting Eq. (5.28) in Eq. (4.43), we obtain

$$
Q_{1}=-\int_{\mu_{0}}^{\mu_{1}}\left[\frac{\mu_{0}}{\sqrt{\mu^{2}-\mu_{1}^{2}}\left(\sqrt{\mu_{0}^{2}-\mu_{1}^{2}}-\sqrt{\mu^{2}-\mu_{1}^{2}}\right)}-\frac{1}{\mu_{0}-\mu}\right] d \mu .
$$

The integrand is now nonsingular at $\mu=\mu_{0}$. We can make this manifest by rationalizing the difference of square roots in the first term. Some simple algebra yields

$$
Q_{1}=-\int_{\mu_{0}}^{\mu_{1}} \frac{1}{\sqrt{\mu^{2}-\mu_{1}^{2}}} \frac{\mu_{0}^{2}+\mu^{2}-\mu_{1}^{2}}{\mu_{0} \sqrt{\mu_{0}^{2}-\mu_{1}^{2}}+\mu \sqrt{\mu^{2}-\mu_{1}^{2}}} d \mu .
$$

We now make the substitution $\mu=\mu_{1} \cosh z$, and define

$$
\cosh z_{0}=\mu_{0} / \mu_{1} \text {. }
$$

This yields

$$
\begin{aligned}
Q_{1} & =-\int_{0}^{z_{0}} \frac{\cosh 2 z_{0}+\cosh 2 z}{\sinh 2 z_{0}+\sinh 2 z} d z \\
& =-\int_{0}^{z_{0}} \frac{\cosh \left(z+z_{0}\right)}{\sinh \left(z+z_{0}\right)} d z=-\ln \left(2 \cosh z_{0}\right) \\
& =-\ln \frac{2 \mu_{0}}{\mu_{1}} .
\end{aligned}
$$

We now have all the ingredients needed to calculate the quantity $F$. Substituting Eqs. (5.23), (5.24), (5.40), and (5.44) in Eq. (4.42), and writing the result in terms of $\lambda$ and $h_{x}$, we obtain Eq. (5.7). Note that in writing down the final answer, we have replaced $\bar{J}$ by $J$ and $h_{x}$ by $h_{x 0}$ in this formula. This is because $F$ is part of the pre-exponential factor in $\Delta_{n}$, which is determined only to leading order in $1 / J$. Keeping higher order corrections by distinguishing between $\bar{J}$ and $J$ or $h_{x}$ and $h_{x 0}$ is not justified.

The final answer (5.5) for $\Delta_{n}$ is obtained by substituting Eqs. (5.8), (5.9), and (5.7) in Eq. (4.39). 


\section{ACKNOWLEDGMENTS}

This work is supported by the NSF via grant number DMR-9616749. I am indebted to Wolfgang Wernsdorfer and Jacques Villain for useful discussions and correspondence about $\mathrm{Fe}_{8}$. 


\section{REFERENCES}

* $\quad$ Electronic address: agarg@northwestern.edu

[1] (a) A. Garg, Europhys. Lett. 22, 205 (1993); (b) Phys. Rev. B 51, 15161 (1995); (c) ibid 60, 6705 (1999); (d) Phys. Rev. Lett. 83, 4385 (1999); (e) submitted to Europhys. Lett.

[2] W. Wernsdorfer and R. Sessoli, Science 284, 133 (1999).

[3] C. Sangregorio, T. Ohm, C. Paulsen, R. Sessoli, and D. Gatteschi, Phys. Rev. Lett. 78, 4645 (1997).

[4] A.-L. Barra, P. Debrunner, D. Gatteschi, Ch. E. Schultz, and R. Sessoli, Europhys. Lett. 35, 133 (1996).

[5] R. Caciuffo, G. Amoretti, A. Murani, R. Sessoli, A. Caneschi, and D. GAtteschi, Phys. Rev. Lett. 81, 4744 (1998).

[6] J. Villain and Anna Fort, submitted to Europhys. B.

[7] G. Herzberg and H. C. Longuet-Higgins, Discuss. Faraday Soc. 35, 77 (1963).

[8] M. V. Berry and M. Wilkinson, Proc. Roy. Soc. Lond. A 392, 15 (1984).

[9] See, e.g., Eqs. (3) and (4) in A. Garg, to appear in Physica B [Proceedings of the XXII International Conference on Low Temperature Physics, Espoo and Helsinki, Finland, Aug. 4-11, 1999]. Or, see Eqs. (5) and (6) of Ref. [1](e).

[10] See Fig. 1 in W. Wernsdorfer, R. Sessoli, A. Caneschi, D. Gatteschi, and A. Cornia, cond-mat/0001346 (unpublished).

[11] R. B. Dingle and J. Morgan, Appl. Sci. Res. 18, 221 (1967); ibid. 18, 238 (1967).

[12] K. Schulten and R. G. Gordon, J. Math. Phys. 16, 1971 (1975).

[13] P. A. Braun, Rev. Mod. Phys. 65, 115 (1993).

[14] J. L. van Hemmen and A. Sütő, (a) Europhys. Lett. 1, 481 (1986); (b) Physica 141B, 37 (1986); (c) in Quantun Tunneling of Magnetization-QTM'94, edited by L. Gunther and B. Barbara (Kluwer, Dordecht, 1995).

[15] A. Garg, submitted to Journal of Mathematical Physics.

[16] Conyers Herring, Rev. Mod. Phys. 34, 631 (1962).

[17] L. P. Gor'kov and L. P.Pitaevski, Dokl. Akad. Nauk SSSR 151, 822 (1963) [English transl.: Soviet Phys.-Dokl. 8, 788 (1964).

[18] S. P. Kou, J. Q. Liang, Y. B. Zhang, and F. C. Pu, Phys. Rev. B 59, 11792 (1999).

[19] J.-Q. Liang, H. J. W. Müller-Kirsten, D. K. Park, and F.-C. Pu, cond-mat/0001142 (unpublished).

[20] J. L. van Hemmen and W. F. Wreszinski, Commun. Math. Phys. 119, 213 (1988).

[21] L. D. Landau and E. M. Lifshitz, Quantum Mechanics (Pergamon, New York, 1977), 3rd ed.. See Sec. 50, Problem 3.

[22] G. Temple, Proc. Roy. Soc. Lond. A 119, 276 (1928).

[23] Tosio Kato, J. Phys. Soc. Jpn. 4, 334 (1949).

[24] A. Garg, J. Math. Phys. 39, 5166 (1998).

[25] In fact, because of the two nearly coincident turning points, it would be necessary to use quadratic connection formulas, given, e.g., by Eqs. (3.63) and (4.21) in M. V. Berry and K. E. Mount, Rep. Prog. Phys. 35, 315 (1972). Use of a linear connection formula, as in Ref. [21], leads to a splitting that lacks the curvature correction factor $g_{n}$ [Eq. (4.10]].

[26] A. Garg, to appear in Amer. J. Phys.

[27] W. H. Furry, Phys. Rev. 71, 360-371 (1947). 
[28] Since the eikonal equation (2.6) has four solutions, there are four DPI solutions, all of which break down at the turning point at $m=-m_{1}$. In Ref. [15] we derive connection formulas for all four solutions, of which, Eq. (4.16) is the case there designated by $\sigma_{1}=-1, \sigma_{2}=1$.

[29] The result in Ref. [21] is missing the correction factor $g_{n}$, as mentioned in footnote [25]. For the ground state this factor is well known and understood, and an elementary discussion is given in Ref. [26].

[30] M. Enz and R. Schilling, J. Phys. C: Solid State 19, L711 (1986).

[31] V. I. Belinicher, C. Providencia, and J. da Providencia, J. Phys. A: Math. Gen. 30, 5633 (1997). 


\section{FIGURES}

FIG. 1. Spectrum of the Hamiltonian (1.1) for $J=3$, as a function of $H_{x} / H_{c} . H_{z} / H_{c}=0$, 0.07454 , and 0.1491 in (a), (b), and (c) respectively. The small ovals indicate points that are narrowly avoided anticrossings, but appear to be crossings on low resolution.

FIG. 2. Critical energy curves for the Hamiltonian (1.1), showing the dual labelling scheme.

FIG. 3. Magnified view of the lower left hand region of Fig. A showing the point of tangency $m^{*}$ between $U_{0}$ and $U_{*}$, and turning points at $m=-m_{t}$ and $-m_{1}$ for an energy $E$.

FIG. 4. Comparison between numerical (solid lines) and analytic [Eqs. (5.5 5.9), dashed lines] results for the splitting between the first three pairs of levels for $H_{z}=0$. The parameters are $k_{1}=0.321 \mathrm{~K}, k_{2}=0.229 \mathrm{~K}$, close to those for $\mathrm{Fe}_{8}$. 

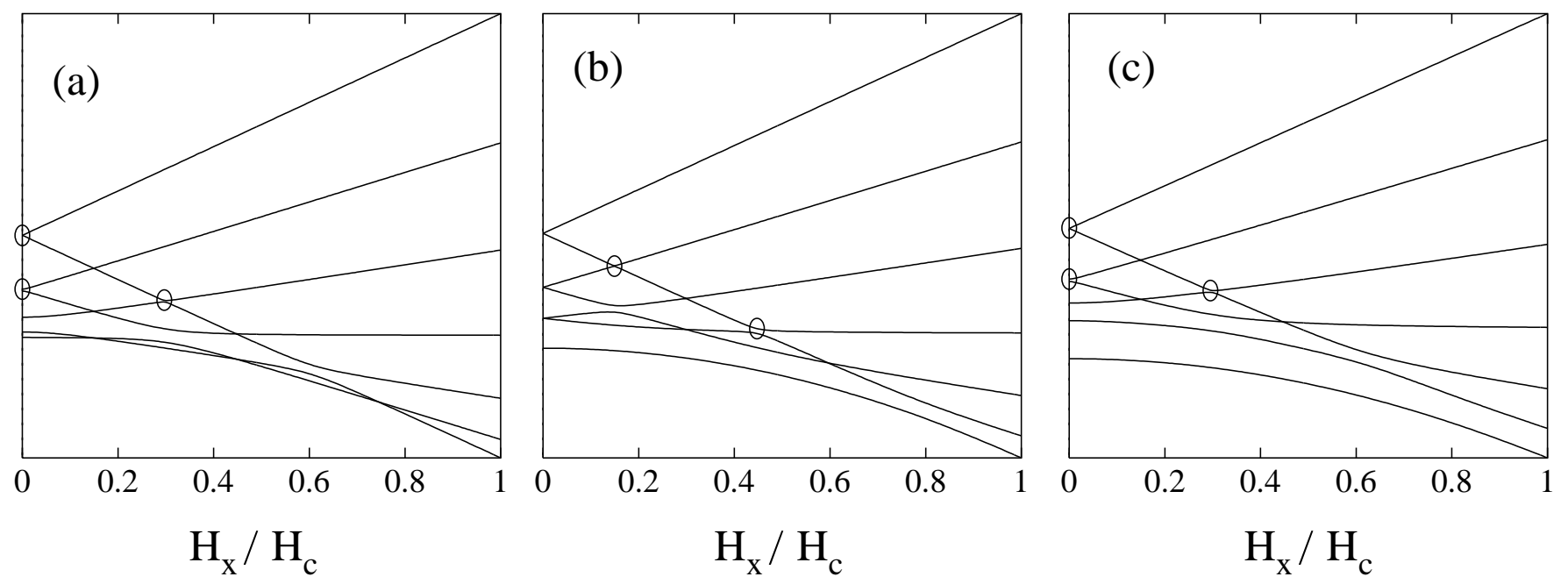


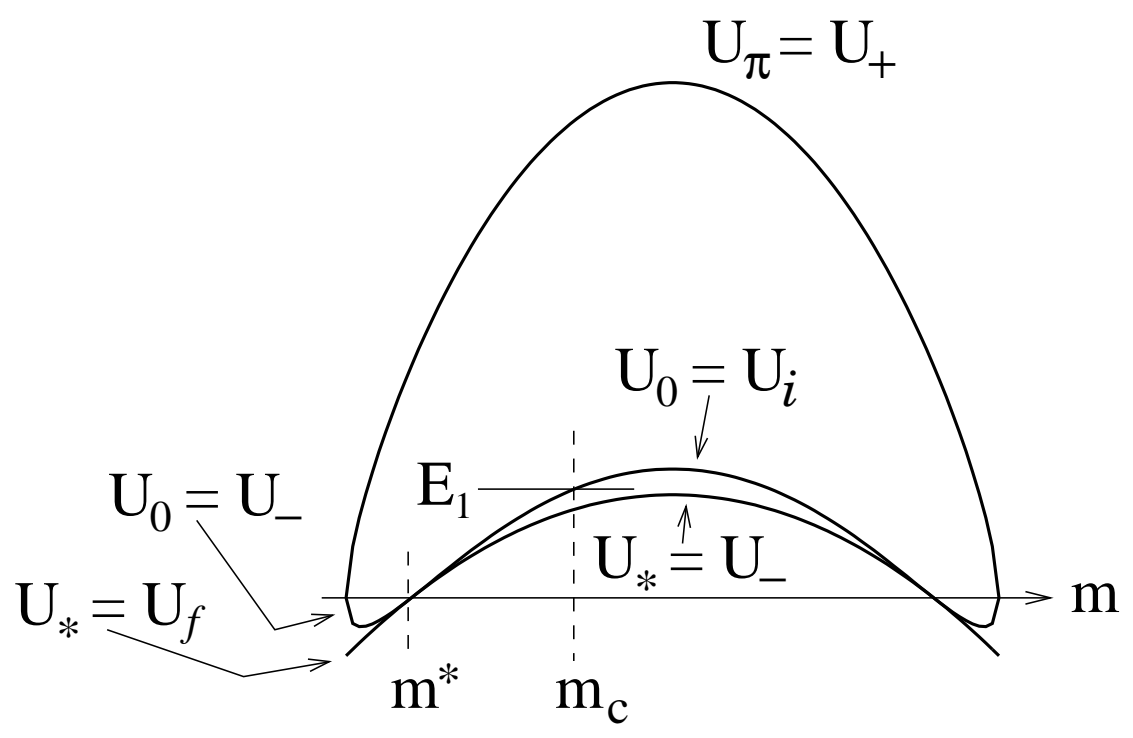




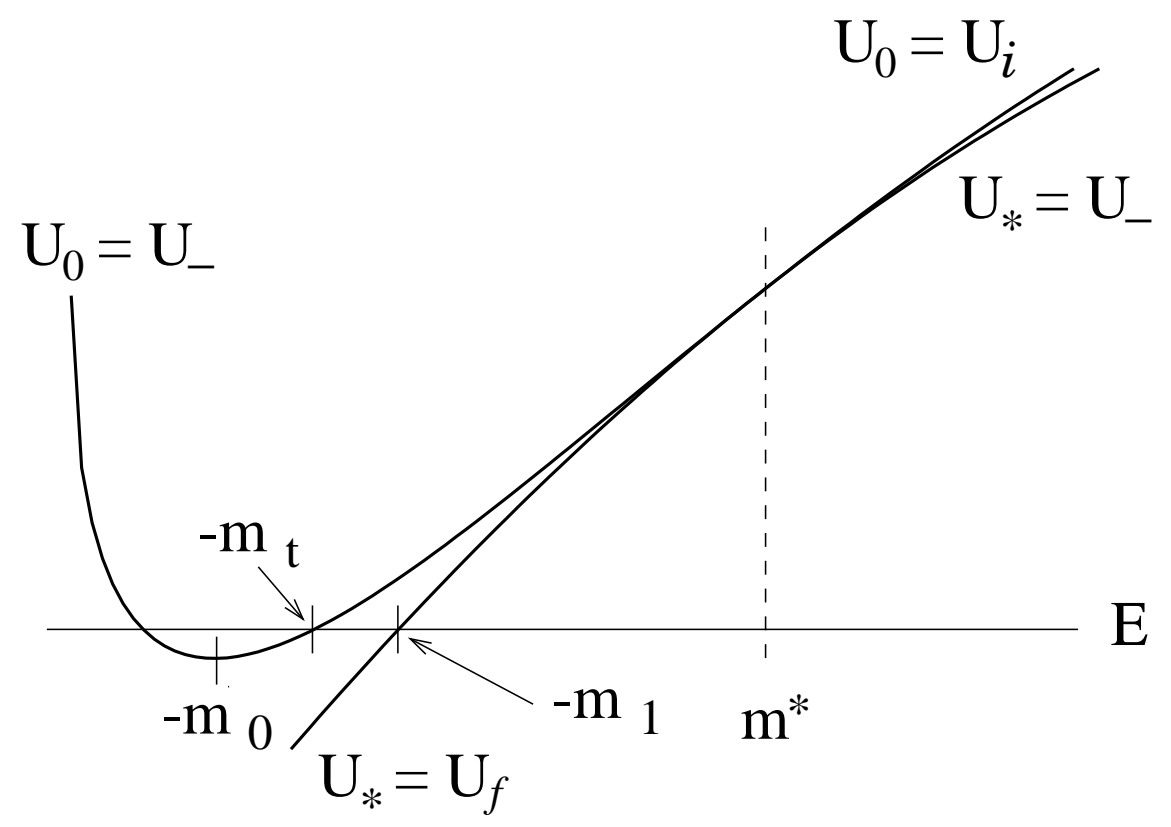




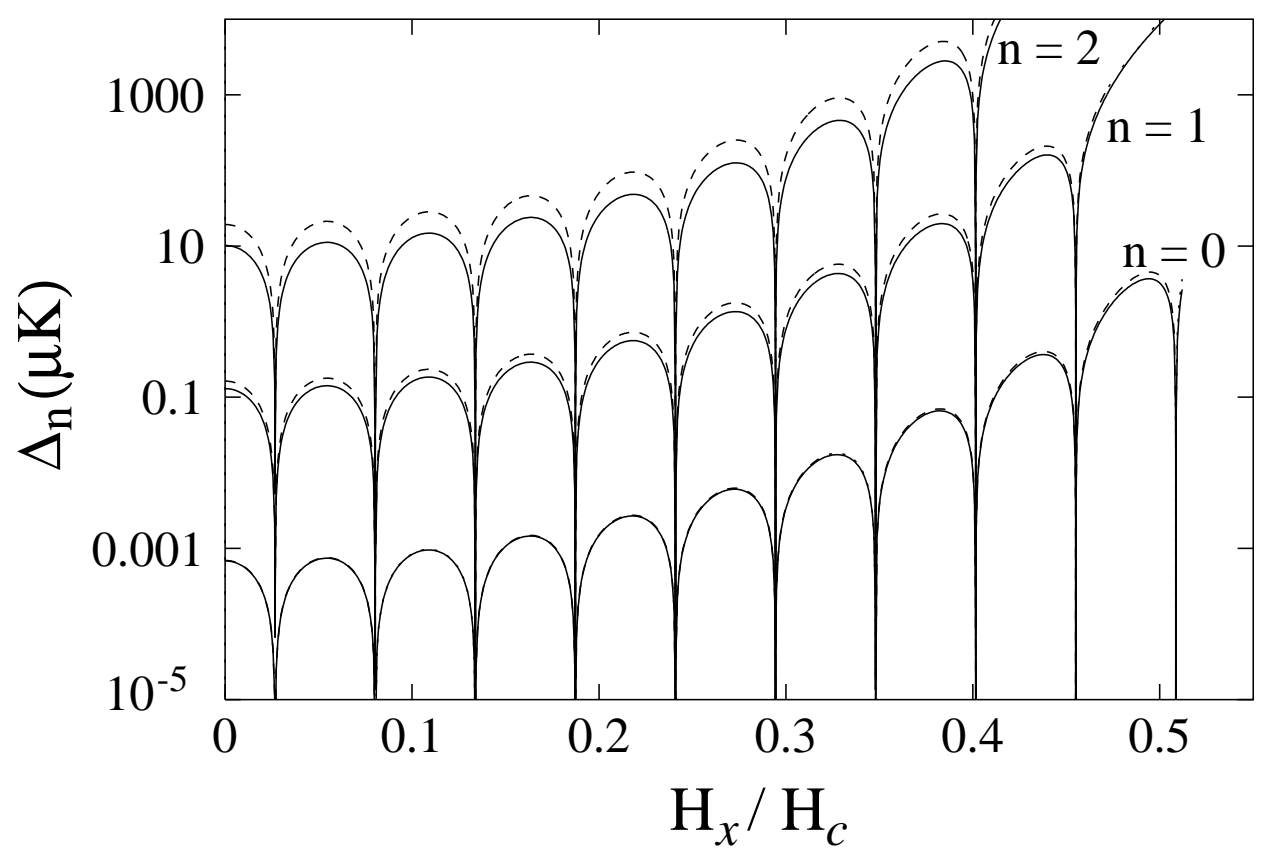

